\title{
Design of Polymer Multimode Racetrack Resonator for Chemical Optical Sensor
}

\author{
Sun-Ho Kim ${ }^{1}$, Su-Jin Jeon ${ }^{1}$, Eudum Kim ${ }^{1}$, Dohyun Kim${ }^{1}$, Kwang Ryong $\mathrm{Oh}^{2}$ and Young-Wan Choi ${ }^{1}$ \\ ${ }^{1}$ School of Electical and Electronics Engineering, Chung-Ang University, 221 Heuksuk-Dong, \\ Dongjak-ku, Seoul, 156-756, Korea \\ ${ }^{2}$ Electronics and Telecommunications Research Institute 138 Gajeongno, Yuseong-Gu, Daejeon, \\ Korea \\ ychoi@cau.ac.kr
}

\begin{abstract}
:
In this paper, the polymer multimode racetrack resonator is simulated for an optical chemical sensor. It consists of the resonator, total internal reflection (TIR) mirrors, and the multimode interference (MMI) coupler. The width and height of the multimode waveguide are designed to be $3 \mu \mathrm{m}$ and $2 \mu \mathrm{m}$. The multimode waveguide is easier to fabricate than a single mode waveguide because of a wide width. In order for a multimode waveguide resonator to operate as an optical sensor, it is necessary to discrimination the higher-order mode. For higher order mode discrimination, we designed novel waveguide with TIR mirrors instead of a straight waveguide at the top of the racetrack resonator. We considered the Goos-Hänchen shift and critical angle when designing a TIR mirrors. As a result of the FDTD simulation, Q-factor of this resonator can be obtained as $1.3 \times 10^{4}$ and the shift of the resonator output peak was $15 \mathrm{~nm} / \mathrm{RIU}$.
\end{abstract}

Key words: Multimode waveguide, polymer resonator, TIR mirror, chemical optical sensor

\section{Introduction}

In recent years, researches on optical resonator sensors used as chemical sensors have been actively conducted $[1,2]$. The resonance wavelength of optical resonator sensor is shifted when a chemical reaction occurs in the resonator sensing region. It can be used as a chemical sensor by detecting resonance wavelength shift. An optical resonator sensor has advantages such as high Q-factor, quick response time, label-free detection and simple geometries [3].

Most ring resonators are made of single mode optical waveguides because multimode waveguide resonator has multiple peaks. For this reason, multimode resonator is difficult to use as a sensor. The width of single mode waveguide is usually smaller than micrometers. This condition serves as a disadvantage of fabrication. We used the multimode waveguide which is easier to fabricate than a single mode waveguide. In addition, we used a MMI coupler that has advantage in fabrication. In this paper, we show that the polymer multimode racetrack resonator can be used as chemical sensors by using the TIR mirrors. The TIR mirrors works to remove multiple peaks for use as a sensor.

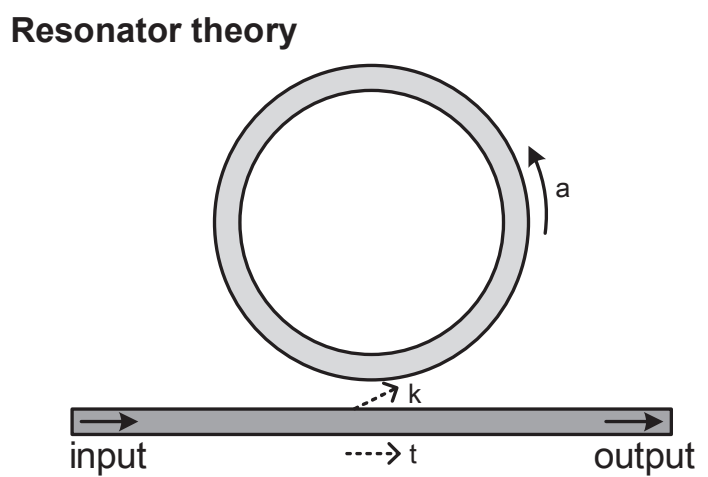

Fig. 1. Basic structure of ring resonator.

The basic structure of resonator is shown in Fig. 1. $E_{i 1}$ is input e-field, $E_{i 2}$ is coupling e-field from resonator to waveguide, $E_{t 1}$ is output e-field, and $E_{\mathrm{t} 2}$ is coupling e-field from waveguide to resonator. The coupling relation equations and output transmission power $\mathrm{P}_{\mathrm{t} 1}$ can be expresses as, [4]

$$
\begin{aligned}
& \left(\begin{array}{l}
E_{t 1} \\
E_{t 2}
\end{array}\right)=\left(\begin{array}{cc}
t & \kappa \\
\kappa^{*} & -t^{*}
\end{array}\right)\left(\begin{array}{l}
E_{i 1} \\
E_{i 2}
\end{array}\right) \\
& \left|t^{2}\right|+\left|\kappa^{2}\right|=1(2)
\end{aligned}
$$


$E_{i 2}=\alpha \cdot e^{j \theta} E_{t 2}$

$P_{t 1}=\frac{\alpha+|t|^{2}-2 \alpha|t| \cos \theta}{1+\alpha^{2}|t|^{2}-2 \alpha|t| \cos \theta}$

$\theta=4 \pi^{2} n_{\text {eff }} \frac{r}{\lambda}$

where $\mathrm{t}$ is the transmission coefficient, $\kappa$ is the coupling coefficient, $\alpha$ is the loss coefficient of the resonator, $r$ is radius of resonator, $n_{\text {eff }}$ is effective refractive index of waveguide, $\lambda$ is wavelength and $\theta$ is phase change of light.

\section{Waveguide and TIR mirror design}

We designed the multimode waveguide which have a SU-8 polymer core and $\mathrm{SiO}_{2}$ cladding. The refractive index of $\mathrm{SU}-8$ polymer and $\mathrm{SiO}_{2}$ are 1.564 and 1.44 at wavelength $1.55 \mu \mathrm{m}$, respectively. The waveguide has a width of 3 $\mu \mathrm{m}$ and a height of $2 \mu \mathrm{m}$. There are 5 TE and TM modes of this waveguide according to the simulation. We considered the Goos-Hänchen shift and critical angle when designing a TIR mirrors. The critical angle is 39.75 degrees. The value of the Goos-Hänchen shift is calculated about $220 \mathrm{~nm}$ when incidence angle is 80 degrees [5]. We designed 160 degrees TIR mirror considering a reflectance of each modes. The higher order mode has a lower effective index of waveguide. Therefore, the reflectance decreases at the same incidence angle. This leads to the effect of eliminating multiple peaks.

\section{Multimode racetrack resonator}

We designed novel waveguide with seven TIR mirrors instead of a straight waveguide at the top of the racetrack resonator for higher order mode discrimination. Figure 3 shows that the simulation results of racetrack normalized resonator output with TIR mirrors. It has resonance peaks with Q-factor of $1.3 \times 10^{4}$ and shift of peak $15 \mathrm{~nm} / \mathrm{RIU}$.

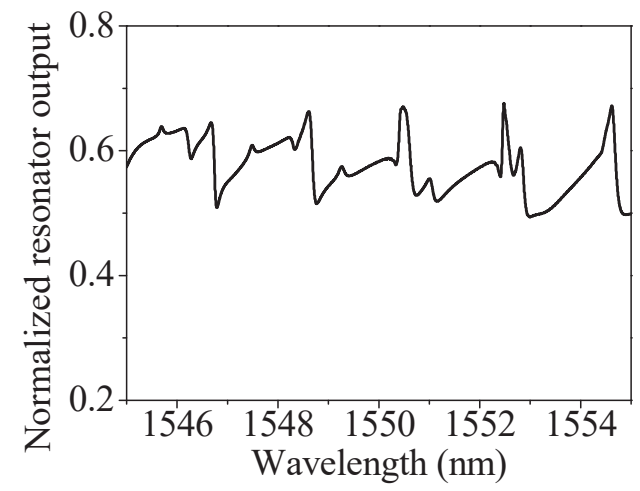

Fig. 2. The output of no TIR mirror resonator.

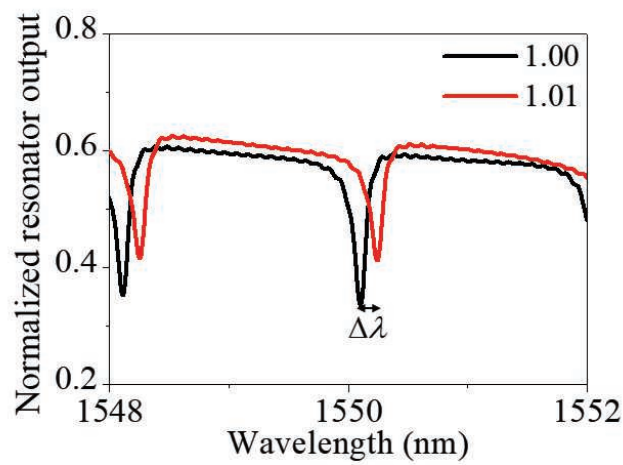

Fig. 3. The output of polymer multimode racetrack resonator with TIR mirror.

\section{Conclusion}

We designed the polymer multimode racetrack resonator with MMI coupler and TIR mirrors. It can used as a sensor since multiple peaks have been removed with TIR mirrors. We plan to optimize the number and angle of the TIR mirror to increase its performance before using it as a chemical sensor.

\section{Acknowledgment}

This research was supported by Basic Science Research Program through the National Research Foundation of Korea (NRF) funded by the Ministry of Education (2009-0093817) and by the Korea Institute for Advancement of Technology (KIAT) grant funded by the Korean government (MOTIE: Ministry of Trade, Industry \& Energy, HRD Program for Software-SoC convergence) (No. N0001883).

\section{Reference}

[1] L. Huang, H. Yan, X. Xu, S. Chakravarty, N. Tang, H. Tian, R. T. Chen, Low detection limit sensor based on subwavelength grating racetrack resonator, Proc. SPIE 10077, Nanoscale Imaging, Sensing, and Actuation for Biomedical Applications XIV, 100770M; doi: 10.1117/12.2252429

[2] J. Su, Label-Free Biological and Chemical Sensing Using Whispering Gallery Mode Optical Resonators: Past, Present, and Future $\mathrm{M}$, Sensors, 17; doi;10.3390

[3] Y. Sun, X. Fan, Optical ring resonators for biochemical and chemical sensing, Analytical and Bioanalytical Chemistry 399, 205-211; doi; 10.1007/s00216-010-4237-z

[4] A. Yariv, Critical Coupling and Its Control in Optical Waveguide-Ring Resonator Systems, IEEE Photonics Technology Letters, 14, 483-485; doi; 10.1109/68.992585

[5] A. W. Snyder, J. D. Love, Goos-Hänchen shift, Applied Optics, 15, 236-238; doi; 10.1364/AO.15.000236 